\title{
Highlighting Differences in Cancer Epidemiology in India: A Descriptive Report from a Private Sector Hospital-Based Cancer Registry in Delhi for the Years 2013 to 2017
}

\author{
Gunjan Shrivastav ${ }^{1}$ Arun Adhana ${ }^{2}$ Kamlesh Kumari ${ }^{2}$ Naseem Khan ${ }^{2}$ Ankit Kumar ${ }^{2}$ \\ Shailender Rathore ${ }^{2}$ Anil Kumar Anand ${ }^{3}$ Ramandeep Singh Arora ${ }^{1}$
}

${ }^{1}$ Department of Medical Oncology, Max Super Speciality Hospital, Saket, New Delhi, India

2Office of Research, Max Super Speciality Hospital, Saket, New Delhi, India

${ }^{3}$ Department of Radiation Oncology, Max Super Speciality Hospital, Saket, New Delhi, India

Ind ] Med Paediatr Oncol 2021;42:346-352.
Address for correspondence Ramandeep Singh Arora, MD, Department of Medical Oncology, Max Super Speciality Hospital, Saket, New Delhi, India 110017 (e-mail: childhoodcancer@gmail.).

\begin{abstract}
\section{Keywords}

- cancer

- epidemiology

- hospital-based cancer registry

- India

- medical tourism

Introduction Cancer continues to grow in number. Knowing the epidemiology helps in prevention and treatment. Existing hospital-based cancer registries (HBCRs) provide little data of private sector including patients for medical tourism.

Objectives The aim of this study was to describe the distribution of cancer at a tertiary center in the private sector in North India for all cancer patients.

Materials and Methods Cases were ascertained from the Oncology Outpatient Department of Max Super Specialty Hospital, Saket, between January 1, 2013, and December 31, 2017, and from pathology database since July 2015. They were abstracted into National Cancer Registry Program (NCRP) core pro forma. We conducted a descriptive analysis of distribution by age, gender, nationality, site, stage, and treatment.

Results Among the 15,850 confirmed cases, 8,034 (51\%) were males. Nearly $1.8 \%$ of patients were pediatric ( $<15$ years) and $30.1 \%$ geriatric ( $>65$ years). A high proportion of patients $(13 \%)$ came from outside India and $27 \%$ from outside Delhi-National Capital Region. The most common cancers in males were prostate (10.9\%), lung (10\%), and mouth (7.6\%). Stomach was the most common site of cancer in international male patients. Four of the top ten cancers in males were of the gastrointestinal tract (esophagus, stomach, colon, and rectum). The most common cancers in females were breast (37.4\%), ovary (7.1\%), and corpus uteri (5.6\%). Tobacco-related cancers accounted for $36.9 \%$ of cancers in males and $11.3 \%$ in females. The composite stage in males was IV in $54 \%$, followed by stage III (21\%), stage II (15\%), and stage I (10\%). In females, stage IV was $33 \%$, stage III was $23 \%$, stage II was $28 \%$, and stage I was $16 \%$. As registry
\end{abstract}

DOI https://doi.org/

$10.1055 / \mathrm{s}-0041-1733820$

ISSN 0971-5851
C 2021. Indian Society of Medical and Paediatric Oncology.

This is an open access article published by Thieme under the terms of the Creative Commons Attribution-NonDerivative-NonCommercial-License, permitting copying and reproduction so long as the original work is given appropriate credit. Contents may not be used for commercial purposes, or adapted, remixed, transformed or built upon. (https://creativecommons.org/licenses/by-nc-nd/4.0/).

Thieme Medical and Scientific Publishers Private Ltd. A-12, Second Floor, Sector -2, NOIDA -201301, India 
included outpatient record visits and pathology records, not all registered patients received treatment at Max Super Specialty Hospital. Overall, $49.8 \%$ of male patients and $49.7 \%$ of female patients received treatment at Max Super Specialty Hospital. Data quality metrics matched other national HBCRs.

Conclusion We highlight the differences from other NCRP HBCRs, with prostate cancer being the top cancer in males and gastrointestinal cancers forming major proportion among all. Socioeconomic status of our patients, referral bias, and international medical tourism could be responsible.

\section{Introduction}

As the population grows and our lifestyle is changing, cancer continues to increase in incidence. As per the International Agency for Research on Cancer, it is estimated that one in five men and one in six women worldwide will develop cancer over the course of their lifetime and that one in eight men and one in 11 women will die from this disease. ${ }^{1}$ Cancer incidence continues to rise in all countries, but as per GLOBOCAN 2018, Asian countries share the burden of nearly half of the new cancer cases and more than half of cancer deaths. The excess burden of deaths is thought to be because of poorer health facilities along with a higher prevalence of cancer types associated with poorer prognosis. By 2025, it is predicted that, worldwide, there will be 20 million new cancer cases, and low- and middle-income countries will share $80 \%$ of the disease burden. ${ }^{2}$

A national health program directed toward cancer screening, prevention, and treatment is increasingly becoming the need of the hour and cancer registries form the very basic foundation for any such endeavor. It was in June 1963 that the first cancer registry program was established in India in Mumbai, ${ }^{3}$ but the program got the requisite boost only in 1982 when the National Cancer Registry Program (NCRP) was established. At present, there are 20 population-based cancer registries (PBCRs) along with 29 hospital-based cancer registries (HBCRs). ${ }^{4}$ While PBCRs provide data on the magnitude, patterns, and trends of disease over time, HBCRs give a better insight regarding the diagnosis and treatment of cancer in patients along with their compliance and long-term follow-up.

Of the $17 \mathrm{HBCRs}$ that were included in the most recent consolidated report of NCRP (2012-2014), ${ }^{5}$ there were only three from North India and only one from the capital city of Delhi. This was from the Dr. B R Ambedkar Institute Rotary Cancer Hospital (BRAIRCH), New Delhi, which is representative of government sector. Private sector has been under represented in the registry data with only 4 out of 17 registries from private institutes, all of which are from southern India. Hence, there are no data on the epidemiology of patients with cancer who seek care in the private sector in North India including patients traveling from other states as well as from outside India to Delhi for medical tourism.
We hereby report the data collected at our tertiary cancer center for all cancer patients registered between 2013 and 2017.

\section{Materials and Methods}

\section{Setting}

Max Healthcare, established in 2000, is a provider of health care to Delhi-National Capital Region (NCR) and North India including cancer care. The oncology division of Max Super Specialty Hospital, Saket, located in South Delhi is one of the hospitals in this network and registers $\sim 3,000$ cancer patients per year.

\section{Case Ascertainment and Registration}

All patients visiting the Oncology Outpatient Record (OPD) of Max Cancer Centre (MCC), Saket, between January 1, 2013, and December 31, 2017, were potentially eligible, and those with cancer were included. The patients were identified using their unique patient identity number. Data were extracted prospectively from the medical record files along with OPD notes, investigation reports, treatment procedures, and discharge summaries. Since July 1, 2015, we have also ascertained cases from the pathology records to enhance the completeness of our registration. This is done by monthly review of all patients with cancer diagnosis in the pathology database, and these are then matched with those which have already been identified through the primary route of MCC OPD. Any new patients identified through this mechanism are then added to the registry database. The data so extracted were abstracted into the NCRP core pro forma. The cancer registry used the International Classification of Diseases, Tenth Revision (ICD-10) version for site classification and ICD-O-3 for histological classification to code the data. The HBCR DM software developed by the NCRP is used for online data entry.

\section{Statistical Analysis}

Data from the HBCR DM software were extracted into Microsoft Excel. We conducted a descriptive analysis of cancer distribution by age, gender, nationality, and stage. Methods of diagnosis were noted for all patients. We studied the distribution of cancer across various sites as 
classified in ICD-10 classification. Site of cancer as per the NCRP nomenclature was further analyzed for individual subgroups, namely, sex (male/female) and geography (native population/foreign patients). Cancers of the lip, tongue, mouth, pharynx (excluding nasopharynx), esophagus, larynx, lung, and urinary bladder were considered as sites of cancer related to tobacco use. We looked into the clinical stage and the extent of disease for all patients and the treatment modalities received.

\section{Ethical Statements}

The institutional ethics committee approved the study with reference number CRP001: NCRP Max Healthcare CT/MSSH/SKT-2/ONCO/12-10. The procedures followed were in accordance with the ethical standards of the responsible committee on human experimentation (institutional) and with the Helsinki Declaration of 1964, as revised in 2013. Written informed consents of patients were taken.

\section{Results}

The total number of patients identified was 24,680 (24,069 through OPD records and additional 611 through pathology records) for the time period 2013 to 2017. Among the registered patients, 15,850 (64.2\%) had cancer and 5,332 (21.6\%) did not have cancer. It was not possible to be certain if the diagnosis was cancer in 3,498 (14.2\%) patients due to inadequate workup or absence of documentation. This category of patients with uncertain diagnosis decreased steadily with time from $43.02 \%$ in January 2013 to $11.30 \%$ in December 2017 as our procedures of patient identification, documentation, and data quality improved. Of the 15,850 patients with confirmed cancer diagnosis, microscopic verification was done in $98.1 \%$ of male patients and $98.4 \%$ of females. The remaining patients were diagnosed mainly on radiology. The flowchart of cancer registry is depicted in - Fig. 1 .

\section{Distribution by Sex, Age, and Geography}

Of the 15,850 cases, 8,034 (51\%) were males and 7,816 (49\%) were females, with a male-to-female ratio of 1:0.97. The age distribution of cancers is seen in - Fig. 2. Data analyzed by 5 -year age groups showed that, among males and females, the peak incidence was seen in the age group of 60 to 64 years. Nearly $1.8 \%$ of cancer patients were pediatric ( $<15$ years age) and $30.1 \%$ were geriatric ( $>65$ years of age). Sixty percent of patients were from Delhi-NCR (South Delhi 38\%, Southwest Delhi 14\%, Gurgaon 8\%, West Delhi 7\%, Northwest Delhi 7\%, East Delhi 5\%, and others 21\%), 27\% from other parts of India, and $13 \%$ from outside India.

\section{Distribution by Site and Variation by Gender and Geography}

The most common sites (organ systems) of cancer in decreasing order were digestive organs (20.7\%); breast (18.9\%); lip, oral cavity, and pharynx (11.8\%); female genital organs (9.5\%); respiratory and intrathoracic organs (9.2\%); lymph, hematological, and related tissues (6.5\%); male genital organs (6.2\%); eye, brain, and central nervous system (4.7\%); urinary tract (3.8\%); melanoma of skin (2.7\%); thyroid and other endocrine glands (1.9\%); ill-defined, secondary, and unspecified sites (1.4\%); bone and articular cartilage (1.4\%); and mesothelial and soft tissue (1.3\%). The breakdown of hematolymphoid malignancies consisted of non-Hodgkin lymphoma (3.1\% of entire population), Hodgkin lymphoma (1.0\% of entire population), leukemia ( $1.6 \%$ of entire population), and multiple myeloma ( $0.9 \%$ of entire population).

The distribution of cancer by site (organs) for males and females is displayed in - Tables $\mathbf{1}$ and 2, respectively. The most common organs for cancer in males by site were prostate $(10.9 \%)$, lung (10\%), and mouth (7.6\%). The number of male patients with other and unspecified sites was $1.5 \%$.

The pattern was slightly different in male patients from outside Delhi where prostate cancer ranked lower than lung

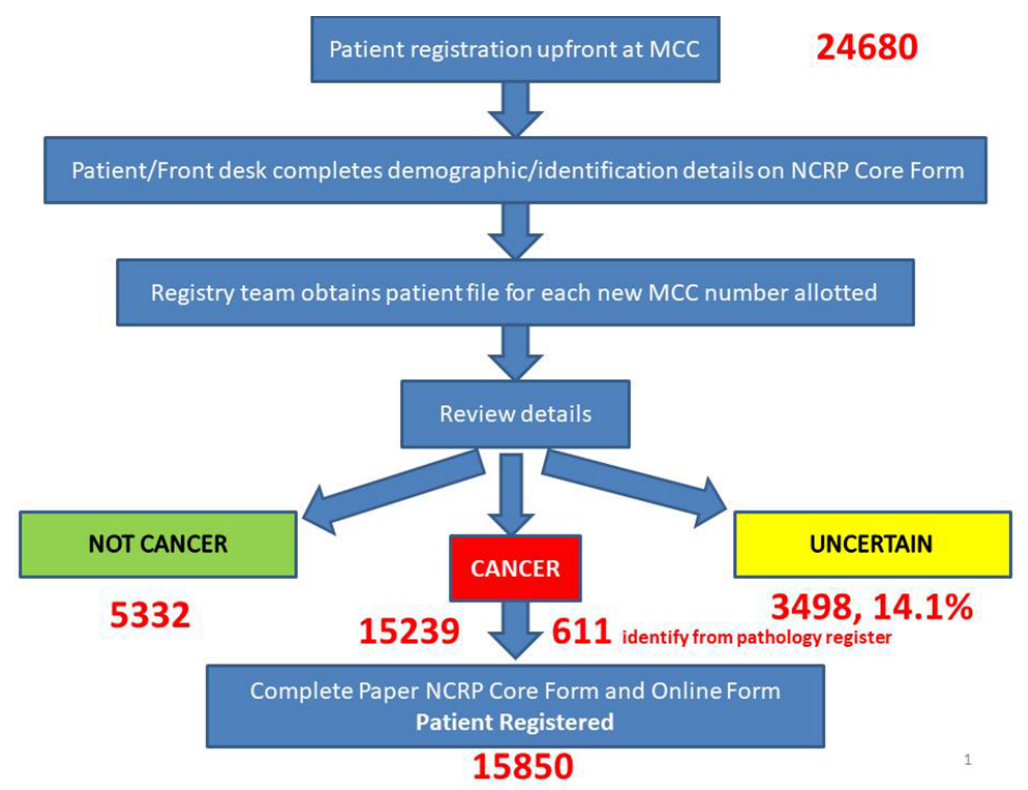

Fig. 1 Flowchart of cancer registry. MCC, Max Cancer Centre; NCRP, National Cancer Registry Program. 


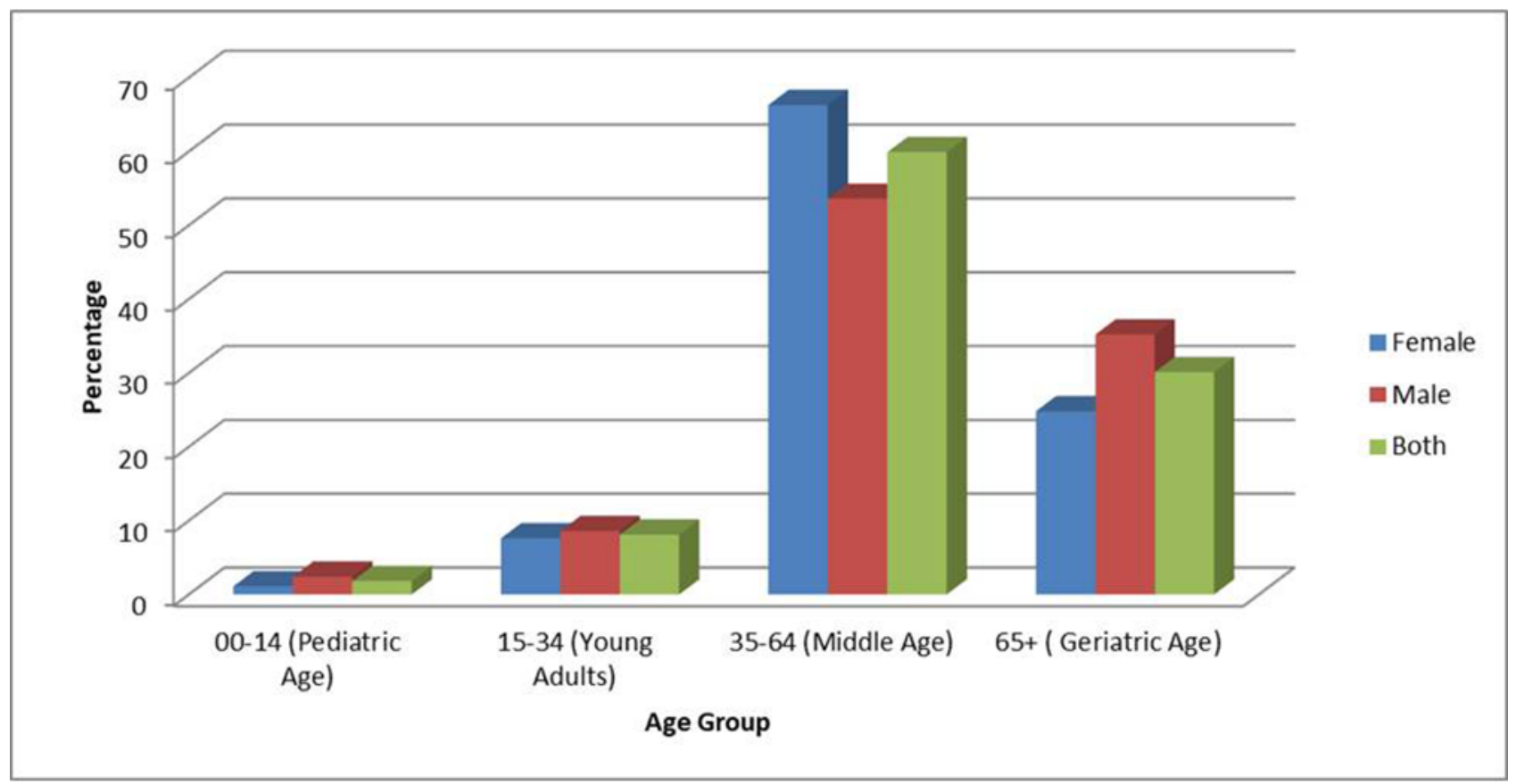

Fig. 2 Distribution of cancer by gender in broad age groups.

Table 1 Top 10 cancer sites by geography in males

\begin{tabular}{|c|c|c|c|c|}
\hline \multirow[t]{2}{*}{ Rank } & \multicolumn{4}{|c|}{ Percentage of all male patients $(n)$} \\
\hline & Overall & Delhi & Outside Delhi (India) & Outside India \\
\hline First & Prostate $(874,10.9 \%)$ & Prostate $(450,5.6 \%)$ & Lung $(341,4.2 \%)$ & Stomach $(113,1.4 \%)$ \\
\hline Second & Lung $(799,9.9 \%)$ & Lung $(374,4.7 \%)$ & Mouth $(338,4.2 \%)$ & $\begin{array}{l}\text { Brain and nervous system } \\
(97,1.2 \%)\end{array}$ \\
\hline Third & Mouth $(614,7.6 \%)$ & Mouth $(259,3.2 \%)$ & Prostate $(335,4.2 \%)$ & Prostate $(89,1.1 \%)$ \\
\hline Fourth & Tongue $(475,5.9 \%)$ & Tongue $(217,2.7 \%)$ & Tongue $(248,3.1 \%)$ & Lung $(84,1.0 \%)$ \\
\hline Fifth & $\begin{array}{l}\text { Brain and nervous system } \\
(445,5.5 \%)\end{array}$ & Esophagus $(174,2.2 \%)$ & $\begin{array}{l}\text { Brain and nervous system } \\
(196,2.4 \%)\end{array}$ & Esophagus $(61,0.8 \%)$ \\
\hline Sixth & Esophagus $(390,4.9 \%)$ & NHL $(162,2.0 \%)$ & Esophagus $(155,1.9 \%)$ & Colon $(52,0.6 \%)$ \\
\hline Seventh & Stomach $(346,4.3 \%)$ & $\begin{array}{l}\text { Brain and nervous system } \\
(152,1.9 \%)\end{array}$ & Colon $(125,1.6 \%)$ & $\mathrm{NHL}(51,0.6 \%)$ \\
\hline Eighth & Colon $(328,4.1 \%)$ & Colon $(151,1.9 \%)$ & Kidney $(120,1.5 \%)$ & Rectum $(51,0.6 \%)$ \\
\hline Ninth & NHL $(326,4.1 \%)$ & Larynx $(133,1.7 \%)$ & $\mathrm{NHL}(112,1.4 \%)$ & Liver $(44,0.5 \%)$ \\
\hline Tenth & Rectum $(265,3.3 \%)$ & Stomach $(123,1.5 \%)$ & Gallbladder $(111,1.4 \%)$ & Bone $(43,0.5 \%)$ \\
\hline
\end{tabular}

Abbreviation: NHL, non-Hodgkin lymphoma.

cancer and mouth cancer. In male patients visiting from outside India, distribution was quite different with cancers of the stomach most commonly followed by brain and nervous system and prostate ( $\mathbf{- T a b l e ~} \mathbf{1}$ ). Cancers of the mouth and tongue did not rank in the top ten cancers in this subset.

The most common organs for cancer in females were breast $(37.4 \%)$, ovary (7.1\%), and corpus uteri (5.6\%). The number of female patients with other and unspecified sites was 25 (1.9\%).

Breast cancer was the most common cancer among all females irrespective of the geography. This was followed by ovary and cervix/uterus in Indian females in contrast to brain and nervous system and cervix in females visiting from outside India ( - Table 2 ).

The top ten cancers in males and females among the four age groups are shown in - Tables 3 and $\mathbf{4}$, respectively.

\section{Tobacco-Related Cancers}

In males, 2,964 tobacco-related cancers (TRCs) were detected, accounting for $36.9 \%$ of all cancer cases. In females, 884 TRCs were detected, accounting for $11.3 \%$ of all cancer cases. Among these cases, lung, esophagus, mouth, tongue, and urinary bladder were the top five sites, in that order (-Table 5). 
Table 2 Top ten cancer sites by geography in females

\begin{tabular}{|c|c|c|c|c|}
\hline \multirow[t]{2}{*}{ Rank } & \multicolumn{4}{|c|}{ Percentage of all female patients $(n)$} \\
\hline & Overall & Delhi & Outside Delhi (India) & Outside India \\
\hline First & Breast $(2926,37.4 \%)$ & Breast $(1517,19.4 \%)$ & Breast $(1115,14.3 \%)$ & Breast $(294,3.8 \%)$ \\
\hline Second & Ovary $(554,7.1 \%)$ & Corpus uteri $(274,3.5 \%)$ & Ovary $(255,3.3 \%)$ & $\begin{array}{l}\text { Brain and nervous system } \\
(67,0.9 \%)\end{array}$ \\
\hline Third & Corpus uteri $(442,5.7 \%)$ & Ovary $(260,3.3 \%)$ & Cervix uteri $(175,2.2 \%)$ & Cervix uteri $(58,0.7 \%)$ \\
\hline Fourth & Cervix uteri $(432,5.5 \%)$ & Cervix uteri $(199,2.5 \%)$ & Gallbladder (174, 2.2\%) & Esophagus $(42,0.5 \%)$ \\
\hline Fifth & Gallbladder $(348,4.5 \%)$ & Gallbladder (157, 2.0\%) & Corpus uteri $(151,1.9 \%)$ & Ovary $(39,0.5 \%)$ \\
\hline Sixth & $\begin{array}{l}\text { Brain and nervous system } \\
(281,3.6 \%)\end{array}$ & Lung $(139,1.8 \%)$ & $\begin{array}{l}\text { Brain and nervous system } \\
(111,1.4 \%)\end{array}$ & Stomach $(34,0.4 \%)$ \\
\hline Seventh & Lung $(269,3.4 \%)$ & $\begin{array}{l}\text { Brain and nervous system } \\
(103,1.3 \%)\end{array}$ & Lung $(104,1.3 \%)$ & Bone $(32,0.4 \%)$ \\
\hline Eighth & Colon $(206,2.6 \%)$ & Colon $(102,1.3 \%)$ & Colon $(83,1.1 \%)$ & Thyroid $(29,0.4 \%)$ \\
\hline Ninth & Esophagus (204, 2.6\%) & Tongue $(87,1.1 \%)$ & Esophagus $(78,1.0 \%)$ & Lung $(26,0.3 \%)$ \\
\hline Tenth & Thyroid (166, 2.1\%) & $\mathrm{NHL}(84,1.1 \%)$ & Mouth $(77,1.0 \%)$ & Rectum $(24,0.3 \%)$ \\
\hline
\end{tabular}

Abbreviation: NHL, non-Hodgkin lymphoma.

Table 3 Top ten cancers by age groups in males

\begin{tabular}{|l|l|l|l|l|}
\hline Rank & $<15$ & $15-34$ & $35-64$ & $>64$ \\
\hline First & Brain and nervous system & Brain and nervous system & Mouth & Prostate \\
\hline Second & Lymphoid leukemia & Bone & Lung, etc. & Lung, etc. \\
\hline Third & Bone & NHL & Tongue & Esophagus \\
\hline Fourth & Hodgkin disease & Hodgkin disease & Prostate & Stomach \\
\hline Fifth & NHL & Testis & Esophagus and nervous system & Mouth \\
\hline Sixth & Connective and soft tissue & Thyroid & Stomach & Colon \\
\hline Seventh & Kidney & Tongue & Colon & Urinary bladder \\
\hline Eighth & Myeloid leukemia & Rectum & NHL & Larynx \\
\hline Ninth & Other skin & Mouth & Kidney & Rectum \\
\hline Tenth & Eye & Other skin & Tongue \\
\hline
\end{tabular}

Abbreviation: NHL, non-Hodgkin lymphoma.

Table 4 Top ten cancers by age groups in females

\begin{tabular}{|l|l|l|l|l|}
\hline Rank & $<15$ & $15-34$ & $35-64$ & $>64$ \\
\hline First & Brain and nervous system & Breast & Breast & Breast \\
\hline Second & Bone & Brain and nervous system & Ovary & Corpus uteri \\
\hline Third & Lymphoid leukemia & Ovary & Cervix uteri & Gallbladder \\
\hline Fourth & Ovary & Thyroid & Corpus uteri & Ovary \\
\hline Fifth & Connective and soft tissue & Bone & Gallbladder & Esophagus \\
\hline Sixth & Kidney & Hodgkin disease & Lung, etc. & Cervix uteri \\
\hline Seventh & Melanoma of skin & NHL & Brain and nervous system & Lung, etc. \\
\hline Eighth & NHL & Colon & Esophagus & Colon \\
\hline Ninth & Eye & Rectum & Colon & NHL \\
\hline Tenth & Liver & Connective and soft tissue & Stomach & Tongue \\
\hline
\end{tabular}

Abbreviation: NHL, non-Hodgkin lymphoma.

\section{Composite Stage}

The composite stage was known in $62 \%$ ( $n=9789$ ) of patients. The majority of the patients in males were stage IV (54\%), followed by stage III (21\%), stage II (15\%), and stage I (10\%). In females, stage IV was proportionally less at $33 \%$, stage III in $23 \%$, stage II in $28 \%$, and stage I in $16 \%$.

\section{Treatment Received}

As registry included OPD visits and pathology records, not all registered patients received treatment at Max Super Specialty Hospital. Overall, $49.8 \%$ of male patients and $49.7 \%$ of female patients received treatment at Max Super Specialty Hospital. A total of 2,757 males received treatment at the reporting 
Table 5 Specific sites of cancer among TRCs

\begin{tabular}{|l|l|l|}
\hline Sites of cancer & Males, $\boldsymbol{n}(\%)$ & Females, $\boldsymbol{n}(\%)$ \\
\hline Lip & $210(7)$ & $5(0.6)$ \\
\hline Tongue & $475(16.0)$ & $161(18.2)$ \\
\hline Mouth & $615(20.7)$ & $161(18.2)$ \\
\hline Oropharynx & $35(1.20)$ & $10(1.1)$ \\
\hline Hypopharynx & $145(4.9)$ & $17(1.9)$ \\
\hline Pharynx & $11(0.4)$ & $1(0.1)$ \\
\hline Esophagus & $390(13.2)$ & $204(23.1)$ \\
\hline Larynx & $248(8.4)$ & $21(2.4)$ \\
\hline Lung & $799(27.0)$ & $269(30.4)$ \\
\hline Urinary bladder & $225(7.6)$ & $35(4.0)$ \\
\hline TRC & $2,964(100)$ & $884(100)$ \\
\hline
\end{tabular}

Abbreviation: TRC, tobacco-related cancer.

institute only. This included single-modality surgery, radiation, and chemotherapy in 823 (29.9\%), 392 (14.2\%), and 631 (22.9\%) patients, respectively. Dual-modality treatment consisted of surgery plus radiation in 118 (4.3\%), surgery + chemotherapy in 92 (3.3\%), and radiation and chemotherapy in $505(18.3 \%)$ patients. Triple-modality treatment was received by $112(4.1 \%)$ males. Hormonal therapy alone or in combination with other modalities was received by $0.9 \%$. The remaining patients received unknown or not documented treatment modality. A total of 3,202 females received treatment at the reporting institute only. This included single-modality surgery, radiation, and chemotherapy in 1,174 (36.7\%), 213 (6.7\%), and 630 (19.7\%), respectively. Dual modality treatment consisted of surgery plus radiation in 160 (5.0\%), surgery + chemotherapy in 439 (13.73\%), and radiation and chemotherapy in $322(10.1 \%)$ patients. Triple modality treatment was received by $174(5.4 \%)$ males. Hormonal therapy alone or in combination with another modality was received by $2 \%$. The remaining patients received unknown or not documented treatment modality.

A total of 1,249 males and 1,320 females received treatment at our institute following prior treatment at any other place. Among males, $77.1 \%$ received single modality and $20.3 \%$ received combination therapy. Among females, $74.2 \%$ received single modality and $23.3 \%$ received combination therapy.

\section{Discussion}

Over the past few years, NCRP has ensured expanding coverage of population covered in cancer registry programs through an increasing number of HBCRs. Despite this, information from North India is relatively sparse and that from the private sector is nonexistent. We provide here a comprehensive registry data of patients coming to our institute over a period of 5 years. The number of patients with uncertain diagnosis has steadily decreased from January 2013 when it was $43.02 \%$ to $11.3 \%$ in December 2017. More than $98 \%$ of patients had a microscopic verification of diagnosis. These data combined with case ascertainment through multiple sources indicate that the quality of registry is high and improving. The number of cancers with sites other and unspecified is $<2 \%$. On external quality checks by NCRP, the number of errors is $2.3 \%$, and this has decreased from $4.7 \%$ in 2013 to $2.2 \%$ in 2017 . This compares favorably with all the HBCRs that were part of the last NCRP report. ${ }^{5}$ Since we have included all patients coming to our OPDs along with data collected from pathologic records, it gives a complete overview of cancer patients presenting to our tertiary cancer center. Unfortunately, the follow-up of all patients was not available, leading to nonavailability of data pertaining to outcomes and survival. This is now an area of focus for our future studies.

Prostate cancer was the most common cancer in males. This is in contrast to registry based at BRAIRCH and many other registries, where carcinoma prostate was not in the top ten common cancers in males. ${ }^{5} \mathrm{~A}$ higher proportion of geriatric patients, a specialized uro-oncology department as well as the socioeconomic status of our patients, could explain the difference. Similarly, four of the top ten cancers in males were from the gastrointestinal tract (esophagus, stomach, colon, and rectum). This was not the case for most other registries except for Amrita Institute, Kochi. ${ }^{5}$ This could represent a tertiary center referral bias for patients requiring specialized surgeries as well as the socioeconomic status of our patients. Furthermore, we saw a difference in the proportion of digestive organs among males of 15 to 34-year age group as against the BRAIRCH registry data. Only HBCRs from Guwahati, Assam, Chennai, and Bangalore see digestive organs among the top ten cancer sites in males of this age group.

In females, breast is now the leading site of cancer across nation accounting for $29.09 \%$ of all cases. The proportion of females with breast cancer in our registry was higher (37.44\%) as compared with BRAIRCH (26.1\%) and also in comparison to private HBCRs from Rajiv Gandhi Cancer Hospital (29.09\%)., ${ }^{5,6}$ As majority of our patient population comes from affluent strata of society, this probably reflects the higher prevalence of breast cancer in high-income groups. The proportion of females with cervical cancer was less in our registry accounting for $5.52 \%$ of all female cancer patients in contrast to BRAIRCH (9.64\%).

One in eight of our patients was from outside India. Data from NCRP HBCR do not provide information on this for other centers, but it is likely that most/all patients in these centers are from India. We also looked at data from Rajiv Gandhi Cancer Hospital, which also operates in the private sector in Delhi-NCR, and although it has no published data as part of the NCRP report, it has made relevant data available on its website. ${ }^{6}$ The proportion of patients registered at our institute from Delhi and surrounding states is comparable with them, but we see a higher proportion of international patients (13\%) as compared with Rajiv Gandhi Cancer Hospital (6\% in 2015). We saw a difference in distribution of cancer between Indian and international patients. Unlike Indian males, prostate cancer was not the most common cancer organ among the international male patients and was surpassed by carcinoma stomach and cancers of the brain and nervous system. This is perhaps due to these cancers requiring specialized surgical 
techniques in their management and has a higher risk of relapse after therapy, requiring second opinions. Probably, due to similar reasons, malignancies of the bones were represented in the top ten cancers among international patients, unlike Indian population.

TRCs accounted for $36.9 \%$ of male cancers and $11.3 \%$ of female cancers, which is comparable to BRAIRCH $(37.2 \%$ in males and $11.0 \%$ in females) but significantly lower than all other cancer registries. ${ }^{5}$ The Government of India has taken leading initiatives to spread awareness against tobacco and related products and for smoking cessation practices. As a result, TRCs are gradually decreasing, but there is still a long way to go. ${ }^{7}$

As an HBCR coming from a private setup and catering to international patients as well, our registry report saw some interesting differences in distribution of cancer as compared with other HBCRs. The distribution of cancer among various age groups was similar with 35 to 64 years old bearing the major burden. Most of the HBCRs from the southern part of the continent list thyroid as one of the top ten sites of cancer in females in the age group of 15 to 34 years, but its contribution decreases in the northern part of the country. BRAIRCH registry does not include thyroid among the top ten cancers, although thyroid ranked 6th in our registry among females of 15 to 34 years' age group.

\section{Conclusion}

Data from our HBCR highlight the differences from previous data reported in the NCRP HBCRs. Key findings include that prostate cancer was the most common site in males and that gastrointestinal cancers formed a much greater proportion of cancers among males and females. These differences could be explained by the higher socioeconomic status of our patients, referral bias for certain specialties, and international medical tourism.

\section{Financial Support and Sponsorship}

Nil.

\section{Conflicts of Interest}

There are no conflicts of interest.

\section{References}

1 Bray F, Znaor A, Cueva P, et al. Planning and Developing Population-Based Cancer Registration in Low- and Middle Income Settings. Lyon, France: IARC Technical Publication; 2014

2 Bray F, Ferlay J, Soerjomataram I, Siegel RL, Torre LA, Jemal A. Global cancer statistics 2018: GLOBOCAN estimates of incidence and mortality worldwide for 36 cancers in 185 countries. CA Cancer J Clin 2018;68(6):394-424

3 Yeole BB, Kurkure AP. An epidemiological assessment of increasing incidence and trends in breast cancer in Mumbai and other sites in India, during the last two decades. Asian Pac J Cancer Prev 2003;4(1):51-56

4 National Centre for Disease Informatics and Research. Consolidated Report of Population Based Cancer Registries, 2012-2014 Bengaluru, India, National Cancer Registry Programme, (NCRP-ICMR). Available from: https://ncdirindia. org/Reports.aspx. Accessed July 11, 2021

5 National Centre for Disease Informatics and Research. Consolidated Report of Hospital Based Cancer Registries, 2012-2014 Bengaluru, India, National Cancer Registry Programme, (NCRP-ICMR). Available from: https://ncdirindia. org/Reports.aspx. Accessed July 11, 2021

6 Hospital Based Cancer Registry Data from 5th Report of Rajiv Gandhi Cancer Institute, Rohini, Delhi. Available from: https:// www.rgcirc.org/wp-content/uploads/2017/09/CancerRegistry-2014-2015.pdf. Accessed July 11, 2021

7 Asthana S, Patil RS, Labani S. Tobacco-related cancers in India: a review of incidence reported from population-based cancer registries. Indian J Med Paediatr Oncol 2016;37(3):152-157 